\title{
Risk factors for neutropenic fever in non- Hodgkin's lymphoma patients with primary granulocyte colony-stimulating factor prophylaxis
}

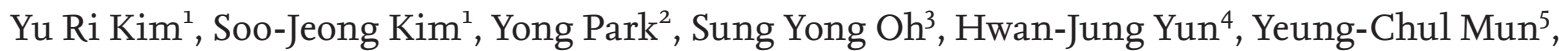 \\ Jin Seok Kim ${ }^{1}$, and the Korean Society of Hematology Lymphoma Working Party
}

\begin{abstract}
${ }^{1}$ Division of Hematology, Department of Internal Medicine, Severance Hospital, Yonsei University College of Medicine, Seoul; ${ }^{2}$ Department of Internal Medicine, Korea University Medical Center, Seoul; ${ }^{3}$ Department of Internal Medicine, Dong-A University College of Medicine, Busan; ${ }^{4}$ Department of Internal Medicine, Chungnam National University College of Medicine, Daejeon; ${ }^{5}$ Department of Internal Medicine, Ewha Womans University School of Medicine, Seoul, Korea
\end{abstract}

Received: May 8, 2020

Revised : June 13, 2020

Accepted: October 29, 2020

Correspondence to

Jin Seok Kim, M.D.

Division of Hematology,

Department of Internal

Medicine, Severance Hospital,

Yonsei University College

of Medicine, 50-1 Yonsei-ro,

Seodaemun-gu, Seoul 03722,

Korea

Tel: +82-2-2228-1972

Fax: +82-2-393-6884

E-mail: hemakim@yuhs.ac https://orcid.org/ooo0-0001$8986-8436$
Background/Aims: Febrile neutropenia (FN) interferes with the proper chemotherapy dose density or intensity in non-Hodgkin's lymphoma (NHL) patients. Chemotherapy with cyclophosphamide, doxorubicin, vincristine, and prednisone $(\mathrm{CHOP}) \pm$ rituximab has an intermediate FN risk. Prophylactic granulocyte colony-stimulating factor (G-CSF) support is recommended for patients with other host-related risk factors.

Methods: We evaluated the risk factors for FN-related admission in NHL patients who have received primary G-CSF (lenograstim) prophylaxis.

Results: Data from 148 patients were analyzed. The incidence of neutropenic fever was 96 events (12.2\%), and the median period was 3.85 days (range, o to 5.9); the median duration of neutropenia was 4.21 days (range, 3.3 to 5.07). Eighty-three FN-related admissions were reported. Advanced age ( $>60$ years), female sex, a low albumin level, and prednisone use were associated with FN-related admission in multivariable analysis $(p=0.010, p<0.001$, and $p=0.010$, respectively). A comparison between diffuse large B-cell lymphoma patients treated with R-CHOP and pegylated G-CSF and those treated with R-CHOP and lenograstim did not reveal significant differences in the FN-related admission rate between the two groups, although the lenograstim-treated group had a higher incidence of severe neutropenia.

Conclusions: Elderly patients, female patients, and patients with low albumin levels need to be actively followed-up for FN even when primary prophylaxis with G-CSF has been used.

Keywords: Febrile neutropenia; Lymphoma, non-Hodgkin; Elderly; Female; Albumin

\section{INTRODUCTION}

Even though a large proportion of non-Hodgkin's lymphoma (NHL) patients could achieve a complete response, chemotherapy-induced febrile neutropenia
(FN) is a major barrier to the completion of the planned chemotherapy. FN is frequently responsible for hospital admission, prolonged hospitalization, and immediate administration of intravenous (IV) antibiotics [1]. It is also associated with increased infection-related 
mortality, with an overall FN-related mortality of $6.8 \%$ to $20 \%[2,3]$. Moreover, it can preclude the delivery of the intended dose of chemotherapeutic agents, interfere with its schedule, or even lead to discontinuation of chemotherapy. Decreasing the dose density or intensity can adversely affect the treatment outcome in NHL [4]. Prevention of chemotherapy-related FN is the most important supportive care for improving treatment outcomes in NHL patients. Therefore, the identification of high-risk patients is of primary relevance in prevention. Although granulocyte colony-stimulating factor (G-CSF) support can help reduce FN-related early deaths and achieve the planned dose and schedules, some patients still experience serious FN-related complications $[5,6]$.

The American Society of Clinical Oncology and the European Organization for Research and Treatment of Cancer recommend primary prophylaxis with G-CSF with a chemotherapy regimen of $\geq 20 \% \mathrm{FN}$ risk or regimens with intermediate FN risk (10\% to $20 \%$ ), wherein the overall risk takes into consideration the patient-related risks $[7,8]$. The FN risk for cyclophosphamide, doxorubicin, vincristine and prednisone (CHOP)-21 \pm rituximab chemotherapy was reported as $17 \%$ to $50 \%[7,9]$, which classifies it as intermediate risk. As previously mentioned, patient-related risk factors should be assessed when using intermediate-risk chemotherapy to assure that prophylactic G-CSF is provided to patients with an overall high risk.

Based on the National Comprehensive Cancer Network guideline, advanced age ( $\geq 65$ years), previous exposure to chemotherapy or radiotherapy, persistent neutropenia, bone marrow involvement of the tumor, poor performance status, recent surgery and/or open wounds, renal or liver dysfunction, and human immunodeficiency virus infection are considered high-risk patient-related factors [10,11]. Among the high-risk patients treated with prophylactic G-CSF, identifying vulnerable populations for FN-related complications is clinically useful for FN prevention and management.

In this study, we evaluated the FN-related outcomes, including FN-related admission, in NHL patients with high-risk patient-related factors treated with CHOP-21 \pm rituximab and prophylactic G-CSF, to identify the predictive factors for FN-related admission.

\section{METHODS}

\section{Patients}

We enrolled 158 patients who were diagnosed with NHL between 2012 and 2015. Ten patients withdrew their consent; therefore, data from 148 patients were analyzed. All patients were scheduled to receive frontline CHOP or R-CHOP chemotherapy. All patients were aged over 20 years and had one or more of the following patient-related factors for FN: age $\geq 65$ years, Ann Arbor stage $\geq$ III, previous chemotherapy or radiation therapy, bone marrow involvement, absolute neutrophil count (ANC) $<1.5$ $\times 10^{9} / \mathrm{L}$ or hemoglobin level $<12.0 \mathrm{~g} / \mathrm{dL}$ before starting chemotherapy, active infection, open wounds, Eastern Cooperative Oncology Group performance status $\geq 2$ or poor nutritional status, hepatic or renal dysfunction, chronic obstructive pulmonary disease, cardiovascular disease, and diabetes mellitus.

\section{Chemotherapy}

All patients were treated with $\mathrm{CHOP} \pm \mathrm{R}$ chemotherapy every 3 weeks. Chemotherapy was composed of cyclophosphamide (750 $\mathrm{mg} / \mathrm{m}^{2}$ IV on day $\mathrm{l}$ ), doxorubicin (50 $\mathrm{mg} / \mathrm{m}^{2} \mathrm{IV}$ on day 1 ), vincristine $\left(1.4 \mathrm{mg} / \mathrm{m}^{2}\right.$ up to $2 \mathrm{mg}$, IV on day 1 ), prednisone (100 $\mathrm{mg}$ oral on day 1 to 5 ), and rituximab $\left(375 \mathrm{mg} / \mathrm{m}^{2} \mathrm{IV}\right.$ on day $\left.\mathrm{1}\right)$. The treatment response was assessed based on the revised International Workshop Criteria [12]. The relative dose intensity (RDI) was calculated as the ratio of the actual dose intensity to the planned dose intensity for a fixed time period $[6,9,13]$ : RDI = actual dose intensity / planned dose intensity; actual dose intensity = actual dose received for each agents $\left(\mathrm{mg} / \mathrm{m}^{2}\right) /$ actual duration of chemotherapy for all agents (days); planned dose intensity = planned total dose for each agent $\left(\mathrm{mg} / \mathrm{m}^{2}\right) /$ planned duration of chemotherapy for all agents (days). Any adverse event was assessed by the National Cancer Institute-Common Terminology Criteria for Adverse Events, 2009 (version 4.0).

\section{Study drug}

Lenograstim (Neutrogin, JW Pharmaceutical Corp., Seoul, Korea) is a recombinant human G-CSF. For prophylaxis, $5 \mu \mathrm{g} / \mathrm{kg} /$ day of lenograstim was injected subcutaneously from days 4 to day 6 after chemotherapy and was continued until the ANC reached $3.0 \times 10^{9} / \mathrm{L}$. In case of outpatients, G-CSF was administered on weekdays 
only, while it was administered continuously without interruption to hospitalized patients. FN was defined as a single oral temperature $\geq 38.3^{\circ} \mathrm{C}$ or a temperature $\geq$ $38.0^{\circ} \mathrm{C}$ sustained for $>1$ hour with an ANC $<0.5 \times 10^{9} / \mathrm{L}$ or $<1.0 \times 10^{9} / \mathrm{L}$ predicted to fall below $0.5 \times 10^{9} / \mathrm{L}$ within 48 hours $[14,15]$.

\section{Statistical analysis}

The primary endpoint of this study was admission associated with FN during chemotherapy. The secondary endpoints were the development of severe neutropenia (defined as ANC < $0.5 \times 10^{9} / \mathrm{L}$ ), duration of neutropenia (defined as ANC $<0.5 \times 10^{9} / \mathrm{L}$ or ANC $<1.0 \times 10^{9} / \mathrm{L}$ ), presence and duration of $\mathrm{FN}$, the incidence and duration of infection, use of IV antibiotics, admission not related to FN, reduction of dose density or intensity, complete response rate, and progression-free survival (PFS) or overall survival (OS). PFS was defined as the time from the study enrollment to the date of disease progression or death from any cause. OS was calculated as the time from the study enrollment to death from any cause or the last follow-up. Treatment outcomes of lenograstim-treated patients were compared with those of pegylated G-CSF treated patients, which were obtained from the historical records. We retrieved the data of patients who completed R-CHOP chemotherapy and received pegylated G-CSF between 2014 and 2019. To adjust for the distribution of baseline characteristics, we matched age, sex, and the international prognostic index. Propensity score matching was performed using a 1:1 matching protocol without replacement. Survival analysis and comparisons were performed using the Kaplan-Meier method and the log-rank test. A logistic regression model was used to calculate odds ratios (ORs) and $95 \%$ confidence intervals (CIs). A p value < 0.05 was considered statistically significant for all analyses. All statistical analyses were performed using SPSS for Windows version 24.0 (IBM Corp., Armonk, NY, USA) and R package version 3.4.4 (R Foundation for Statistical Computing, Vienna, Austria).

\section{Ethical statement}

The study protocol was approved by the Institutional Review Board of each participating institution (Severance Hospital IRB No. 4-2011-0432). Informed consent was obtained from all individual participants included in the study.

\section{RESULTS}

\section{Patients' characteristics}

Baseline characteristics are shown in Table 1. Median age was 69 years (range, 24 to 83 ) with 89 (60.1\%) male patients. All patients had at least one risk factor for FN before chemotherapy: four (2.7\%), previous chemotherapy; two (1.4\%), previous radiation; $14(9.5 \%)$, bone marrow involvement at diagnosis; 4 (2.7\%), low ANC (< 1.5 $\left.\times 10^{9} / \mathrm{L}\right) ; 63$ (42.6\%), hemoglobin level below $12.0 \mathrm{~g} / \mathrm{dL}$, 34 (23.0\%), low albumin level (<3.5 g/dL); four (2.7\%), infections; one (0.7\%), open wound; eight (5.4\%), renal impairment; 14 (9.5\%), hepatic disease; one (0.7\%), chronic obstructive pulmonary disease; 37 (25.0\%), cardiovascular disease; 23 (15.5\%), diabetes mellitus; 43 (29.1\%), hy-

Table 1. Baseline characteristics

\begin{tabular}{|c|c|}
\hline Variable & No. of patients (\%) \\
\hline \multicolumn{2}{|l|}{ Age, yr } \\
\hline$\leq 60$ & $51(34.5)$ \\
\hline$>60$ & $97(65.5)$ \\
\hline \multicolumn{2}{|l|}{ Sex } \\
\hline Male & $89(60.1)$ \\
\hline Female & $59(39.9)$ \\
\hline \multicolumn{2}{|c|}{ ECOG performance status } \\
\hline $0-1$ & $135(91.2)$ \\
\hline$\geq 2$ & $13(8.8)$ \\
\hline \multicolumn{2}{|l|}{ Stage } \\
\hline I/II & $67(45 \cdot 3)$ \\
\hline III/IV & $81(54.7)$ \\
\hline \multicolumn{2}{|c|}{ Extranodal involvement } \\
\hline $0-1$ & $87(58.8)$ \\
\hline$>1$ & $61(41.2)$ \\
\hline \multicolumn{2}{|l|}{ LDH levels } \\
\hline Reference & $62(41.9)$ \\
\hline Elevated & $86(58.1)$ \\
\hline \multicolumn{2}{|l|}{ IPI } \\
\hline Low & $50(33.8)$ \\
\hline Low intermediate & $27(18.2)$ \\
\hline High intermediate & $39(26.4)$ \\
\hline High & $32(21.6)$ \\
\hline
\end{tabular}


Table 2. Predictive factors for FN-associated admission in the lenograstim-treated group

\begin{tabular}{|c|c|c|c|}
\hline Univariable analysis & Odd ratio & $95 \% \mathrm{CI}$ & $p$ value \\
\hline Age $>60$ years & 2.761 & $1.215-6.273$ & $0.015^{\mathrm{a}}$ \\
\hline Female sex & 4.947 & $2.365-10.352$ & $<0.001^{\mathrm{a}}$ \\
\hline $\mathrm{ECOG}>2$ & 1.120 & $0.366-3.454$ & 0.843 \\
\hline Stage III/IV & 1.232 & $0.615-2.467$ & 0.600 \\
\hline Extranodal involvement > 1 site & 1.417 & $0.709-2.829$ & 0.324 \\
\hline Elevated LDH & 1.426 & $0.705-2.886$ & 0.323 \\
\hline Body surface area & 5.050 & $0.698-37.037$ & 0.108 \\
\hline Bone marrow involvement & 1.244 & $0.401-3.862$ & 0.706 \\
\hline Previous chemotherapy/radiotherapy & 1.493 & $0.342-6.522$ & 0.594 \\
\hline Active infection & 0.807 & $0.082-7.970$ & 0.855 \\
\hline Renal dysfunction & 1.233 & $0.295-5.153$ & 0.775 \\
\hline Hepatic dysfunction & 0.874 & $0.263-2.902$ & 0.827 \\
\hline Chronic obstructive lung disease & NA & & \\
\hline Cardiovascular disease & 2.064 & $0.965-4.415$ & 0.062 \\
\hline Anemia $<12.0 \mathrm{~g} / \mathrm{dL}$ & 1.268 & $0.636-2.526$ & 0.500 \\
\hline Low albumin & 2.625 & $1.271-5.421$ & $0.009^{\mathrm{a}}$ \\
\hline Neutropenia $<1.5 \times 10^{9} / \mathrm{L}$ & NA & & \\
\hline Lymphopenia $<1 \times 10^{9} / \mathrm{L}$ & 1.977 & $0.811-4.826$ & 0.134 \\
\hline Rituximab use & 0.607 & $0.219-1.678$ & 0.336 \\
\hline RDI-cyclophosphamide & 0.100 & $0.013-0.773$ & $0.027^{\mathrm{a}}$ \\
\hline RDI-doxorubicin & 0.152 & $0.021-1.123$ & 0.065 \\
\hline RDI-vincristine & 0.611 & $0.119-3.123$ & 0.554 \\
\hline RDI-prednisone & 0.031 & $0.003-0.354$ & $0.005^{\mathrm{a}}$ \\
\hline \multicolumn{4}{|l|}{ Multivariable analysis } \\
\hline Age $>60$ years & $4 \cdot 925$ & $1.681-14.435$ & $0.004^{a}$ \\
\hline Female sex & $5 \cdot 747$ & $2.341-14.084$ & $<0.001^{\mathrm{a}}$ \\
\hline Low albumin & 3.073 & $1.244-7.587$ & $0.015^{\mathrm{a}}$ \\
\hline
\end{tabular}

FN, febrile neutropenia; CI, confidence interval; ECOG, Eastern Cooperative Oncology Group; LDH, lactate dehydrogenase; NA, not available; RDI, relative dose intensity.

${ }^{\mathrm{a}}$ Statistically significant.

pertension; 12 (8.1\%), hepatitis; and seven (4.7\%), history of tuberculosis.

\section{Treatment outcomes}

Median follow-up duration was 10.0 months (range, o to 38). The OS was $84.0 \%$ and PFS was $74.0 \%$ at 1-year. Among the 109 patients who completed the planned chemotherapy, 86 (78.9\%) achieved complete response, 14 (12.8\%) achieved partial response, five (4.6\%) had stable disease, and four (3.7\%) had progressive disease. The 
Table 3. Baseline characteristics in each group of patients after propensity score matching

\begin{tabular}{lccc}
\hline Variable & Lenograstim & Pegylated filgrastim & $p$ value \\
\hline Age, yr, median (range) & $65(24-83)$ & $65(24-86)$ & 0.238 \\
Sex, male/female & $36 / 61$ & $35 / 62$ & 0.999 \\
ECOG O-1/ 2 & $89 / 8$ & $92 / 5$ & 0.568 \\
Stage I or II/ III or IV & $49 / 48$ & $51 / 46$ & 0.886 \\
Extranodal involvement O-1/>1 & $58 / 39$ & $69 / 28$ & 0.131 \\
LDH, normal/elevated & $41 / 56$ & $45 / 52$ & 0.665 \\
IPI & & & \\
$\quad$ Low/low intermediate & $39 / 15$ & $32 / 17$ & 0.696 \\
\hline High intermediate/high & $26 / 17$ & $26 / 22$ & \\
\hline
\end{tabular}

ECOG, Eastern Cooperative Oncology Group; LDH, lactate dehydrogenase; IPI, international prognostic index.

RDIs of the chemotherapeutic agents were as follows: rituximab 0.95 (0.38 to 1.02), cyclophosphamide 0.89 (0.32 to 1.18 ), doxorubicin 0.86 (0.34 to 1.02$)$, vincristine 0.73 (0.27 to 1.05), and prednisone 0.96 (0.20 to 1.35). Throughout the chemotherapy cycles, in total, 775 G-CSF were administered and the median number of G-CSF used was 5 per cycle (range, 1 to 30 ).

\section{Safety}

The safety profiles of lenograstim were assessed by adverse events. These results were assessed as secondary endpoints. Severe neutropenia (ANC < $0.5 \times 10^{9} / \mathrm{L}$ ) was observed in 322 events among the 786 chemotherapy cycles, and the median duration of neutropenia was 4.21 days (range, 3.3 to 5.07). The incidence of FN was 96 events (12.2\%); the median duration of FN was 3.85 days (range, o to 5.9). The number of cases of FN-related infection was 103; 95 patients received IV antibiotics, and the median duration of IV antibiotics was 8.5 days (range, 7.2 to 15). In total, 90 admissions were reported, of which 83 were related to FN, while the rest were due to general weakness, and infection without neutropenia, among other causes.

\section{Predictive factors for FN-related admission}

The primary endpoint of this study was FN-associated admission during chemotherapy. The total number of FN-associated admissions was 83 (10.5\%) among 786 cycles of chemotherapy. Three factors were significantly associated with FN-related admission on multivariable analysis (Table 2): advanced age ( $>60$ years) (OR, 2.761;
95\% CI, 1.1215 to $6.273 ; p=0.015$ ), female sex (OR, 4.947; 95\% CI, 2.365 to 10.352; $p<0.001$ ), and low albumin level (OR; $2.625 ; 95 \%$ CI, 1.271 to $5.421 ; p=0.009$ ). Other risk factors such as poor performance status $(p=0.843)$, advanced disease stage $(p=0.600)$, extranodal involvement at more than one site $(p=0.324)$, elevated lactic dehydrogenase levels $(p=0.323)$, lymphopenia $<1 \times 10^{9} / \mathrm{L}(p$ $=0.134)$, and the use of rituximab $(p=0.336)$ were not related with $\mathrm{FN}$-associated admission. Among patients who received R-CHOP chemotherapy, advanced age (> 60 years) (OR, 4.730; 95\% CI, 1.468 to $15.241 ; p=0.009)$ and female sex (OR, 3.782; 95\% CI, 1.506 to 9.501; $p=0.005$ ) were predictive factors for FN-related admission.

\section{Comparison with pegylated G-CSF-treated patients}

A total of 97 patients were included in each group using propensity score matching (Table 3). The total number of FN-related admissions was not different between the two groups (47 events in the lenograstim group and 37 events in the pegylated G-CSF-treated group; $p=0.451$ ). The incidence of severe neutropenia was 39.3\% (226 events of 575 cycles) in the lenograstim-treated group and $18.5 \%$ (105 events among 567 cycles) in the pegylated G-CSF-treated group $(p<0.001)$. The duration of neutropenia during the first cycle was 4.59 days in the lenograstim-treated group and 2.78 days in the pegylated G-CSF-treated group $(p<0.001)$. Other secondary endpoints were not different (Table 4 ).

OS rate was $98.0 \%$ in the lenograstim-treated group and $94.0 \%$ in the control group at 1 year $(p=0.535)$ (Fig. IA). PFS rate was $89.0 \%$ in lenograstim-treated group 
Table 4. Secondary endpoints of each group of patients after propensity score matching

\begin{tabular}{lccc}
\hline Variable & Lenograstim & Pegylated filgrastim & $p$ value \\
\hline FN-related admission & 47 & 38 & 0.451 \\
Severe neutropenia & $226 / 575$ & $105 / 567$ & $<0.001$ \\
Duration of neutropenia (1st cycle), day & 4.59 & 2.78 & 47 \\
Incidence of FN & 51 & $3.0(1.0-5.5)$ & 0.001 \\
Duration of FN (1st cycle), day & $4.37(3.11-5.92)$ & 7 & 0.171 \\
Microbial infection & 1 & 46 & 0.065 \\
IV antibiotics & 44 & $8.0(7.0-11.0)$ & 0.495 \\
Duration of IV antibiotics (1st cycle), day & $8.58(7.25-15)$ & 66 & 0.176 \\
Number of admissions & 61 & 0.227 & \\
\hline
\end{tabular}

Values are presented as median (range).

FN, febrile neutropenia; IV, intravenous.
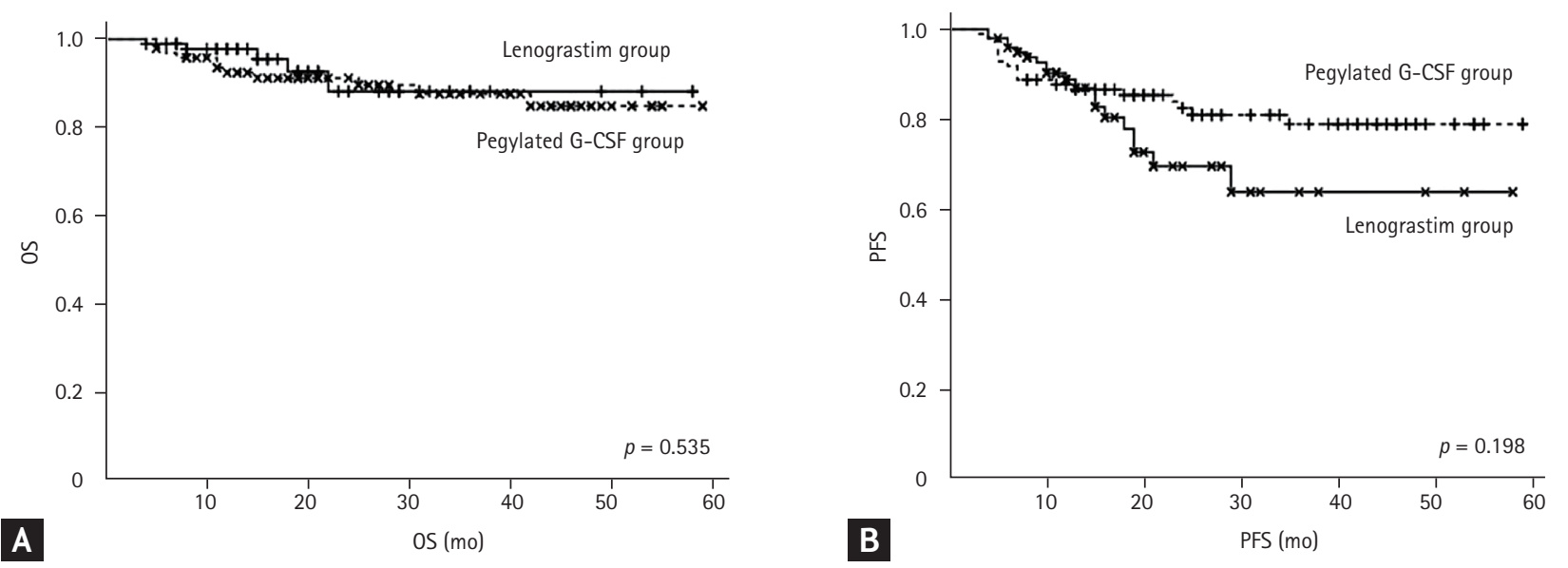

Figure 1. (A) Overall survival (OS) and (B) progression-free survival (PFS) of patients according to the type of granulocyte colony-stimulating factor (G-CSF) support.

and $87.0 \%$ in the pegylated G-CSF-treated group at 1 year $(p=0.198)$ (Fig. 1B). The RDI of rituximab, cyclophosphamide, doxorubicin, and vincristine were higher in the pegylated G-CSF-treated group $(p=0.001, p=$ $0.001, p<0.001$, and $p<0.001)$; the RDI of prednisone was not different $(p=0.422)$.

\section{DISCUSSION}

This multicenter prospective observational study evaluated the FN-related outcomes of primary G-CSF support in patients who received $\mathrm{CHOP} \pm \mathrm{R}$ chemotherapy.
The primary endpoint of this study was the FN-related admission rate, which was found to be $10.5 \%$. Advanced age, female sex, and low albumin levels were associated with FN-related admission. Compared with pegylated G-CSF-treated patients as a historical control, primary prophylaxis with lenograstim showed non-inferior FN related admission rate, although the incidence of severe neutropenia was higher in the lenograstim group.

The chemotherapy regimen, patient-related factors, and tumor type and burden should be considered as the risk factors for FN [10]. All enrolled patients had at least one patient-related risk factor for FN, and we evaluated the predictive factors for $\mathrm{FN}$-associated admis- 
sion among these patients. The incidence of FN was 96 events of 786 cycles (12.2\%), and the FN-related admission rate was $10 \%$. This value was lower than that previously reported $[7,9]$. Among lymphoma patients treated with CHOP-like chemotherapy without primary G-CSF prophylaxis, the FN-related admission rate was reported to be $17 \%$ to $50 \%[9,16]$. Prophylactic G-CSF initiated in the first chemotherapy cycle was associated with a 0.84 time lower risk of FN [5]. The most common adverse reaction to G-CSF administration was musculoskeletal pain, which could be managed with analgesics [17]. In this study, toxicity was mainly related to chemotherapy.

Age $\geq 65$ years, low albumin level, neutropenia, presence of hepatic disease, planned average RDI $>80 \%$, and no early G-CSF use increased the initial hospitalization rate [9]. We used prophylactic G-CSF support in all highrisk patients; however, advanced age, low albumin level, and female sex still had statistical significance with respect to FN-related admission. The RDI of rituximab, cyclophosphamide, and prednisone were risk factors for FN-related admission on univariable analysis but it failed to show statistical significance on multivariable analysis.

Age is an established risk factor for FN, and advanced age has been related to serious complications, including death [18-20]. The cut-off value for age is usually 65 years, although 60 years has been used in some reports [21]. In this study, 60 years of age was a significant risk factor for FN that requires admission. Albumin levels have been known to be related to FN and reflect the nutritional status of cancer patients [22]. Female sex is also a predictive factor for FN [9,17]. Advanced age, female sex, and low albumin level led to a 4.9-, 5.7-, and 3.0- times increase in the risk for FN-related admission in patients with prophylactic support, respectively. Therefore, more aggressive approaches and supportive care are needed to prevent FN-related admission in patients with these risk factors.

After propensity score matching, there were no differences in FN-related admissions between the lenograstim and pegylated G-CSF groups. Although the incidence of severe neutropenia was higher in the lenograstim group, this could be because blood test was not done regularly in pegylated G-CSF group. Response rate was not different between the groups, although the chemotherapy RDI was higher in the pegylated G-CSF group. Pegylated G-CSF could be superior in maintaining a consistent dose density, and observation that needs further investigation. Finally, long-term follow-up is required to reveal the relation between dose density and treatment outcome. Previous studies revealed that four single doses of filgrastim are equivalent to pegylated filgrastim in terms of maintaining the dose intensity of an R-CHOP regimen administered every 2 weeks [23,24]. According to this study, a daily dose of $5 \mu \mathrm{g} / \mathrm{kg}$ lenograstim until ANC recovery or one dose of $6 \mathrm{mg}$ pegylated G-CSF can work as treatment options for NHL patients with patient-related risk factors.

This study has some limitations. We used daily G-CSF for NHL patients instead of pegylated G-CSF, which is now widely used for primary prophylaxis with $\mathrm{CHOP} \pm$ R chemotherapy for NHL. At the beginning of the study, prophylactic pegylated G-CSF was not widely used in Korea. Moreover, it was usually used for secondary prophylaxis after neutropenia development. Therefore, we compared the efficacy of daily primary prophylactic use of G-CSF and prophylactic use of pegylated G-CSF using propensity score matching from historical control. Furthermore, the follow-up period was not sufficiently long, and there was no relation between dose intensity and treatment outcome. Long-term follow-up is needed to elucidate this issue. This study was significant in terms of determining the predictive factors for FN-related admission in high-risk patients who receive CHOP$21 \pm \mathrm{R}$ chemotherapy and primary prophylactic G-CSF support. Most importantly, the final goal of R-CHOP chemotherapy in diffuse large B-cell lymphoma was not palliative but curative; therefore, reducing the infection-related complications during chemotherapy is very important.

In conclusion, this study showed the FN-related outcomes of primary G-CSF prophylaxis in NHL patients with high-risk patient-related factors. Patients with advanced age, female sex, or low albumin levels before chemotherapy should be managed cautiously to minimize the risk of FN-related admission despite early primary prophylactic G-CSF support. 


\section{KEY MESSAGE}

1. Febrile neutropenia (FN)-related admission rate was not different between non-Hodgkin's lymphoma patients who received primary granulocyte colony-stimulating factor (G-CSF) prophylaxis and those who received pegylated G-CSF prophylaxis.

2. Elderly patients, female patients and patients with low albumin levels need to be actively followed-up for FN.

\section{Conflict of interest}

This study was sponsored by JW Pharmaceutical (Seoul, Korea).

\section{REFERENCES}

1. Dale DC, McCarter GC, Crawford J, Lyman GH. Myelotoxicity and dose intensity of chemotherapy: reporting practices from randomized clinical trials. J Natl Compr Canc Netw 2003;1:440-454.

2. Kuderer NM, Dale DC, Crawford J, Cosler LE, Lyman GH. Mortality, morbidity, and cost associated with febrile neutropenia in adult cancer patients. Cancer 2006;106:22582266.

3. Lyman GH, Michels SL, Reynolds MW, Barron R, Tomic $\mathrm{KS}, \mathrm{Yu}$ J. Risk of mortality in patients with cancer who experience febrile neutropenia. Cancer 2010;116:5555-5563.

4. Kwak LW, Halpern J, Olshen RA, Horning SJ. Prognostic significance of actual dose intensity in diffuse large-cell lymphoma: results of a tree-structured survival analysis. J Clin Oncol 1990;8:963-977.

5. Lyman GH, Dale DC, Friedberg J, Crawford J, Fisher RI. Incidence and predictors of low chemotherapy dose-intensity in aggressive non-Hodgkin's lymphoma: a nationwide study. J Clin Oncol 2004;22:4302-4311.

6. Yamaguchi H, Hirakawa T, Inokuchi K. Importance of relative dose intensity in chemotherapy for diffuse large B-cell lymphoma. J Clin Exp Hematop 2011;51:1-5.

7. Aapro MS, Bohlius J, Cameron DA, et al. 2010 Update of EORTC guidelines for the use of granulocyte-colony stimulating factor to reduce the incidence of chemotherapy-induced febrile neutropenia in adult patients with lymphoproliferative disorders and solid tumours. Eur J Cancer 2011;47:8-32.

8. Smith TJ, Bohlke K, Lyman GH, et al. Recommendations for the use of WBC growth factors: American Society of Clinical Oncology Clinical Practice Guideline Update. J Clin Oncol 2015;33:3199-3212.

9. Lyman GH, Delgado DJ. Risk and timing of hospitalization for febrile neutropenia in patients receiving CHOP, CHOP-R, or CNOP chemotherapy for intermediate-grade non-Hodgkin lymphoma. Cancer 2003;98:2402-2409.

10. Lyman GH, Abella E, Pettengell R. Risk factors for febrile neutropenia among patients with cancer receiving chemotherapy: a systematic review. Crit Rev Oncol Hematol 2014;90:190-199.

11. National Comprehensive Cancer Network. Hematopoietic growth factors (version 1.0, 2020) [Internet]. Plymouth Meeting (PA): NCCN, 2020 [cited 2021 Jun 30]. Available from: https://www.nccn.org/professionals/physician_gls/ pdf/growthfactors.pdf.

12. Cheson BD, Pfistner B, Juweid ME, et al. Revised response criteria for malignant lymphoma. J Clin Oncol 2007;25:579-586.

13. Hryniuk W, Bush H. The importance of dose intensity in chemotherapy of metastatic breast cancer. J Clin Oncol 1984;2:1281-1288.

14. Crawford J, Caserta C, Roila F; ESMO Guidelines Working Group. Hematopoietic growth factors: ESMO clinical practice guidelines for the applications. Ann Oncol 2010;21 Suppl 5:v248-v251.

15. Lyman GH, Kuderer NM. Epidemiology of febrile neutropenia. Support Cancer Ther 2003;1:23-35.

16. Osby E, Hagberg H, Kvaloy S, et al. CHOP is superior to CNOP in elderly patients with aggressive lymphoma while outcome is unaffected by filgrastim treatment: results of a Nordic Lymphoma Group randomized trial. Blood 2003;101:3840-3848.

17. Kuderer NM, Dale DC, Crawford J, Lyman GH. Impact of primary prophylaxis with granulocyte colony-stimulating factor on febrile neutropenia and mortality in adult cancer patients receiving chemotherapy: a systematic review. J Clin Oncol 2007;25:3158-3167.

18. Talcott JA, Siegel RD, Finberg R, Goldman L. Risk assessment in cancer patients with fever and neutropenia: a prospective, two-center validation of a prediction rule. J Clin Oncol 1992;10:316-322.

19. Salar A, Haioun C, Rossi FG, et al. The need for improved 
neutropenia risk assessment in DLBCL patients receiving R-CHOP-21: findings from clinical practice. Leuk Res 2012;36:548-553.

20. Pettengell R, Bosly A, Szucs TD, et al. Multivariate analysis of febrile neutropenia occurrence in patients with non-Hodgkin lymphoma: data from the INC-EU Prospective Observational European Neutropenia Study. Br J Haematol 2009;144:677-685.

21. Laskey RA, Poniewierski MS, Lopez MA, et al. Predictors of severe and febrile neutropenia during primary chemotherapy for ovarian cancer. Gynecol Oncol 2012;125:625630.

22. Intragumtornchai $T$, Sutheesophon J, Sutcharitchan $P$, Swasdikul D. A predictive model for life-threatening neutropenia and febrile neutropenia after the first course of CHOP chemotherapy in patients with aggressive non-Hodgkin's lymphoma. Leuk Lymphoma 2000;37:351360.

23. Bozzoli V, Tisi MC, Maiolo E, et al. Four doses of unpegylated versus one dose of pegylated filgrastim as supportive therapy in R-CHOP-14 for elderly patients with diffuse large B-cell lymphoma. Br J Haematol 2015;169:787-794.

24. Kubo K, Miyazaki Y, Murayama T, et al. A randomized, double-blind trial of pegfilgrastim versus filgrastim for the management of neutropenia during $\operatorname{CHASE}(\mathrm{R})$ chemotherapy for malignant lymphoma. Br J Haematol 2016;174:563-570. 